\title{
Erratum to: Development of suitable standards for quantitative determination of persimmon phenol contents in Folin-Ciocalteu and vanillin assays
}

Bo Zou • Xiao-qian Dong $\cdot$ Zhen-zhen Ge $\cdot$ Ze Xu

Jing Du $\cdot$ Chun-mei Li

Published online: 28 June 2014

(C) Springer-Verlag Berlin Heidelberg 2014

Erratum to: Eur Food Res Technol

DOI 10.1007/s00217-014-2232-x

In the original publication, the corresponding author was wrongly published as Dr. Bo Zou. The correct corresponding author for this article is "Dr. Chun-mei Li".

The online version of the original article can be found under doi:10.1007/s00217-014-2232-x.

B. Zou $\cdot$ X. Dong $\cdot$ Z. Ge $\cdot$ Z. Xu $\cdot$ J. Du $\cdot$ C. Li

College of Food Science and Technology, Huazhong Agricultural

University, Wuhan 430070, China

e-mail: skzoubo@163.com

C. $\operatorname{Li}(\bowtie)$

Key Laboratory of Environment Correlative Food Science,

Ministry of Education, Huazhong Agricultural University,

Wuhan, China

e-mail: lichmyl@mail.hzau.edu.cn 